\title{
Editorial: Adiabatic Quantum Computation
}

\author{
Jacob D. Biamonte* \\ Deep Quantum Laboratory, Skolkovo Institute of Science and Technology, Moscow, Russia
}

Keywords: adiabatic quantum computing, universality, adiabatic error correction, experimental demonstrations, graph and switching circuit embeddings, gadget Hamiltonians, adiabatic theorem, Hamiltonian complexity theory

\author{
Editorial on the Research Topic
}

\section{Adiabatic Quantum Computation}

\section{INTRODUCTION}

Around the time when this special issue was proposed (circa 2014) the quantum computing community began to more widely consider the potential of quantum enhanced annealing. At the time, it was not so trendy as it is today. And a venue was desirable for dissemination of results into the scientific record. I felt I could help facilitate this for two reasons. The first was that I had worked on the topic for some time so was both aware and supportive of its development. The second was that I was at the time not actively working on the specific topic, meaning I felt I could judge the significance of the submissions as a bit of an outsider with insider knowledge.

The interest at that time was on the technology being developed by D-Wave Systems Inc. While these devices are not universal quantum computers, they remain an interesting class of quantum enhanced annealing devices. To this day, many of the central open questions considered in the special issue still remain. However, the six papers appearing in the issue all did make some impact. I will now discuss the accepted papers in the order which they were published and very briefly highlight the progress they made.

Edited and reviewed by: Alex Hansen Norwegian University of Science and Technology, Norway

*Correspondence: Jacob D. Biamonte j.biamonte@skoltech.ru

Specialty section: This article was submitted to Interdisciplinary Physics, a section of the journal Frontiers in Physics

Received: 17 August 2019 Accepted: 27 August 2019 Published: 12 September 2019

Citation:

Biamonte JD (2019) Editorial: Adiabatic Quantum Computation. Front. Phys. 7:130.

doi: 10.3389/fphy.2019.00130

\section{THE SIX ACCEPTED PIECES}

The first published piece was "Adiabatic quantum optimization for associative memory recall” by Hadayat Seddiqi and Travis S. Humble of Oak Ridge National Laboratory Seddiqi and Humble. The paper was among the first to merge ideas from machine learning with adiabatic quantum annealing. Indeed, the article formulated the recall of memories stored in a Hopfield network using energy minimization by adiabatic quantum optimization Seddiqi and Humble. As of today, according to Google Scholar the article has received seventeen citations with the topic of the article just as relevant-if not more so-today.

The second paper to appear Bian et al., "Discrete optimization using quantum annealing on sparse Ising models" by the authors representing a team out of D-Wave Systems Inc. in alphabetical order Zhengbing Bian, Fabian Chudak, Robert Israel, Brad Lackey, William G. Macready and Aidan Roy has made rather wide impact. According to Google Scholar, as of today the article has reached a total of 63 citations. Several techniques presented in the article related to problem instance embedding to the Ising model Bian et al. The techniques remain relevant.

The third paper Smolin and Smith generated wide interest and even controversy as the community tried to understand the role of quantum effects in these new devices. The paper titled, 
"Classical signature of quantum annealing" by John A. Smolin and Graeme Smith has also received 63 citations as of today according to Google Scholar.

The forth paper to appear Hen on the futuristic topic, "Fourier-transforming with quantum annealers." The paper considered the prospects of using quantum adiabatic evolution to execute the quantum Fourier transform, a key building block of many quantum algorithms.

The fifth paper to appear, "How to test the quantumness of a quantum computer?" was by Alexandre M. Zagoskin1, Evgeni Il'ichev, Miroslav Grajcar, Joseph J. Betouras and Franco Nori. The article appeared as an interesting perspective, discussing how to verify the quantum properties of the recently introduced quantum annealing devices by D-Wave Systems Inc. According to Google Scholar, the article Zagoskin et al. has been cited 25 times and counting.

The sixth and final article to appear was last but certainly not least. It was by Andrew Lucas, at the time he was finishing his doctorate at Harvard. The article Lucas was titled "Ising formulations of many NP problems" and with 370 citations according to Google Scholar as of today, has emerged as a classic in the field. The article provided Ising formulations for many NP-complete and NP-hard problems, including all of Karp's 21 NP-complete problems. It was published as a review.

\section{CLOSING REMARKS}

Now five years later, the data paints a picture we would not have anticipated. Of the six published articles, the work by Lucas has emerged as a classic. With two of the other six papers also remaining highly relevant today. I believe that with the new interest in quantum enhanced machine learning, the early work Seddiqi and Humble using Hopfield networks stands to have still more impact. According to Google Scholar, as of today the six published articles received over 542 citations. Although citations are not always the best judge of significance, in this case they clearly show impact.

\section{THE CALL FOR SUBMISSIONS}

Adiabatic Quantum Computing (AQC) relies on the idea of embedding a problem into a physical system, such that the systems lowest energy configuration stores the problems solution. While any quantum algorithm can be run on a universal adiabatic quantum computer in principle, combinatorial optimization problems appear to be the most natural for near-term devices. Recent experimental progress has resulted in annealers with around 500 spins and studying the quantum properties of these devices has sparked dramatic interest and scientific debate in this rapidly growing area. More generally, ground state computation unites computer science and physics by studying the computational complexity of evolving a system into its ground state. Theoretical and experimental papers as well as detailed numerical investigations related to adiabatic quantum computation and quantum annealing are sought.

\section{AUTHOR CONTRIBUTIONS}

The author confirms being the sole contributor of this work and has approved it for publication.

\section{ACKNOWLEDGMENTS}

JB thanks the authors for submitting high quality research and for engaging professionally and actively in the Frontiers online review system.

Conflict of Interest Statement: The author declares that the research was conducted in the absence of any commercial or financial relationships that could be construed as a potential conflict of interest.

Copyright $\odot 2019$ Biamonte. This is an open-access article distributed under the terms of the Creative Commons Attribution License (CC BY). The use, distribution or reproduction in other forums is permitted, provided the original author(s) and the copyright owner(s) are credited and that the original publication in this journal is cited, in accordance with accepted academic practice. No use, distribution or reproduction is permitted which does not comply with these terms. 